\title{
Diphthamide Biosynthesis 1 is a Novel Oncogene in Colorectal Cancer Cells and is Regulated by MiR-218-5p
}

\author{
Minghui Liu ${ }^{a}$ Kai Yinn ${ }^{a, b}, c \quad X u$ Guo ${ }^{a}$ Huijin Feng ${ }^{a}$ Min Yuan ${ }^{a}$ Yanqing Liu \\ Jianguo Zhang ${ }^{d}$ Baoliang Guo ${ }^{d}$ Chen Wanga Guangxin Zhou ${ }^{\mathrm{e}}$ Zhen Zhou ${ }^{\mathrm{a}}$ \\ Chen-Yu Zhang ${ }^{\mathrm{a}} \quad$ Xi Chen ${ }^{\mathrm{a}}$
}

\begin{abstract}
aState Key Laboratory of Pharmaceutical Biotechnology, Jiangsu Engineering Research Center for MicroRNA Biology and Biotechnology, NJU Advanced Institute for Life Sciences (NAILS), School of Life Sciences, Nanjing University, ${ }^{b}$ Department of Gastrointestinal Surgery, Nanjing Drum Tower Hospital Clinical College of Nanjing Medical University, Nanjing, Jiangsu, 'Department of General Surgery, Taixing Hospital Affiliated to Bengbu Medical School, Taixing, Jiangsu, 'The Second Affiliated Hospital of Harbin Medical University, Harbin, Heilongjiang, 'Department of Orthopedics, Jinling Hospital, School of Medicine, Nanjing University, Nanjing, Jiangsu, China
\end{abstract}

\section{Key Words}

Dph1 • MiR-218-5p • Tumourigenesis $•$ CRC

\begin{abstract}
Background/Aims: This study focused on the oncogenic role of Diphthamide biosynthesis 1 (DPH1) in colorectal cancer (CRC) cells. Methods: The expression of DPH1 was determined by quantitative RT-PCR analysis and western blotting in CRC tissues. The role of DPH1 in CRC cells was investigated via cell viability and invasion assays under the condition of DPH1 silencing or overexpression. Bioinformatics analysis and luciferase reporter analysis were used to identify the upstream microRNA which might regulate DPH1.The inverse correlation between the microRNA and DPH1 was also detected in CRC cells. Results: We identified an unexpected role for DPH1 as an oncogene in CRC cells. The tumour-suppressive miR-218-5p regulates $\mathrm{DPH} 1$ directly and negatively. Loss of miR-218-5p drives the oncogenic role of DPH1 in CRC cells. Conclusion: The modulation of DPH1 by miR-218-5p may be an important regulatory axis during CRCtumourigenesis.
\end{abstract}

\section{Introduction}

According to cancer statistics, CRC is both the third most common form of cancer and the third most frequent cause of cancer deaths in the USA[1]. In China, colorectal cancer is one of the most frequently diagnosed cancers, amounting for $9.2 \%$ of all new cancer cases

M. Liu, K. Yin and X. Guo contributed equally to this work. 


\section{Cellular Physiology \\ \begin{tabular}{l|l|l} 
and Biochemistry Published online: November 17, 2017 & $\begin{array}{l}\text { (c) } 2017 \text { The Author(s). Published by S. Karger AG, Basel } \\
\text { www.karger.com/cpb }\end{array}$ \\
\hline
\end{tabular} \\ Liu et al.: DPH1 Functions as an Oncogene in CRC Cells and is Regulated by miR-218-5p}

and $7.09 \%$ of all cancer deaths [2]. Although decreasing colorectal cancer mortality has been observed worldwide in recent years, the incidence trend has been increasing. Therefore, it is still necessary to investigate the pathogenesis of CRCand develop more effective prevention strategies and therapeutics for patients with CRC.

Diphthamide biosynthesis 1 (DPH1), also known as OVCA1 (ovarian cancer-associated gene 1), encodes an enzyme that performs posttranslational modifications on translation elongation factor 2 (EF2) in the biosynthesis of diphthamide in eukaryotes [3]. DPH1 used to be considered a tumour suppressor gene because its deficiency correlates with the development of both ovarian and breast cancer $[4,5]$. However, in the last decade, there have been few reports on the tumour suppressive role of DPH1 in other tumour phenotypes. In this article, we investigated the expression pattern of DPH1 in human CRC tissues. Unexpectedly, we found that DPH1 protein levels were upregulated in CRC tissues, suggesting that DPH1 may play a different role in CRC.

microRNAs (miRNAs) are small single-stranded non-coding RNA molecules that typically reduce the translation and stability of mRNAs by targeting the $3^{\prime}$ untranslated region (3'UTR) of mRNAs [6]. miRNAs play significant roles in many cell functions including cell cycle, proliferation and invasion. Dysregulation of miRNA expression can lead to pathogeneses such as cancer [7]. miR-218 is a well-known tumour suppressor in a number of cancers, including CRC $[8,9]$, lung cancer [10] and breast cancer [11]. In CRC, miR-218 was reported to inhibit cell cycle and to promote apoptosis [12]. Decreased miR-218 expression has been reported to correlate with poor prognosis in CRC patients [9]. However, the underlying molecular mechanism of the contribution of miR-218 to CRC tumorigenesis is not fully understood.

In this paper, we validated that DPH1 functions as an oncogene in CRC cells. We next experimentally confirmed the negative regulation of DPH1 by miR-218-5p in CRC cells. We also showed that loss of miR-218-5p expression leads to an increase in DPH1 in CRC, which in turn promotes cell proliferation and invasion in CRC cells.

\section{Materials and Methods}

\section{Human tissues and cells}

The colorectal cancer tissues (T) and their noncancerous tissues (N) were obtained from the Affiliated Gulou Hospital of Nanjing University (Nanjing, China). Tissues fragments were transported in liquid nitrogen and stored at $-80^{\circ} \mathrm{C}$. The patients approved this study. Table 1 listed all the patients' clinical features. The Ethics Committee signed written informed consent. SW480 and HT29 cells were cultured in RPMI 1640 containing 10\% foetal bovine serum (FBS, Gibco, Carlsbad, CA, USA) at $37^{\circ} \mathrm{C} / 5 \%$ $\mathrm{CO}_{2}$.

Isolation of RNA and quantitative RT-PCR

Total RNA was isolated according to the manufacturer's instructions using the TRIzol reagent (Sigma, St. Louis, MO, USA). Quantitative RT-PCR was performed to quantify miRNA levels and mRNA levels (see our previous study for details [24]).The primers for PCR amplifications

Table 1.Clinical features of colorectal cancer patients

\begin{tabular}{lcccc}
\hline Case No. & Gender & Age & Tumor subtype & Pathological Stage \\
\hline 1 & Male & 73 & Adenocarcinoma & II \\
2 & Male & 58 & Adenocarcinoma & II \\
3 & Female & 65 & Adenocarcinoma & III \\
4 & Male & 63 & Adenocarcinoma & III \\
5 & Female & 67 & Adenocarcinoma & II \\
6 & Female & 59 & Adenocarcinoma & III \\
7 & Male & 80 & Adenocarcinoma & IV \\
8 & Male & 48 & Adenocarcinoma & III \\
9 & Male & 67 & Adenocarcinoma & II \\
10 & Female & 66 & Adenocarcinoma & III \\
11 & Male & 46 & Adenocarcinoma & III \\
12 & Male & 50 & Adenocarcinoma & IV \\
13 & Male & 71 & Adenocarcinoma & III \\
\hline
\end{tabular}




\section{Cellular Physiology Cell Physiol Biochem 2017;44:505-514 \begin{tabular}{ll|l} 
and Biochemistry & $\begin{array}{l}\text { DOI: } 10.1159 / 000485087 \\
\text { Published online: November 17, } 2017\end{array}$ & $\begin{array}{l}\text { C } 2017 \text { The Author(s). Published by S. Karger AG, Basel } \\
\text { www.karger.com/cpb }\end{array}$ \\
\hline
\end{tabular}}

Liu et al.: DPH1 Functions as an Oncogene in CRC Cells and is Regulated by miR-218-5p

were as follows: DPH1 (forward primer), 5'-CTGAAAGCCGAGTATCGTGTG-3'; DPH1 (reverse primer) 5'-TGTTCTCTGGATAGGACTTTGCT-3'; GAPDH (sense), 5'-CGAGCCACATCGCTCAGACA-3'; and GAPDH (antisense), 5'-GTGGTGAAGACGCCAGTGGA-3'. GAPDH and U6 were used as internal reference genes for mRNA and miRNA quantifications, respectively.

\section{Protein extraction and Western blotting}

Protein was extracted from cells or tissues using ice-cold RIPA lysis buffer (Beyotime, Shanghai, China) with freshly added PMSF (Beyotime, Shanghai, China). The concentration was quantified via Pierce BCA protein assay kit (Thermo Scientific, Rockford, IL, USA). The expression patterns of DPH1 were assessed by Western blotting with DPH1 antibody (ab185960, Abcam's RabMAb technology, USA), and normalized to GAPDH (sc-25778, Santa Cruz Bio-technology, USA).

\section{miRNA overexpression or knockdown}

Cells were transfected with a synthetic RNA oligonucleotide duplex mimicking the miRNA precursor to overexpress miRNA. For this article, it is called pre-miR-218-5p. A synthetic, single-stranded antisense oligonucleotide targeting the mature miRNA was used to knock down miRNA. For this article, it is called anti-miR-218-5p. Synthetic RNA molecules were purchased from RiboBio (Guangzhou, China). Seeded on 12-well plates, cells were transfected with RNA duplexes using Lipofectamine 2000 (Invitrogen) according to the manufacturer's guidelines. The medium was changed to 2\% FBS 1640 after $6 \mathrm{~h}$. Cells were collected at $24 \mathrm{~h}$ and $48 \mathrm{~h}$ after transfection for RNA isolation and protein extraction, respectively.

\section{SiRNA interference assay and plasmid construction}

The siRNA sequences (RiboBio) were as follows: DPH1 siRNA-1:5'-AGGGCGCCAGATCAGACTT-3' (sense); DPH1 siRNA-2: 5'-GTGCATTGTGCTTCCGCTC-3' (sense); DPH1 siRNA-3: 5'-GCCGAGCTCGTGTGCCTCA-3' (sense). A plasmid (Realgene, Nanjing, China) encoding the full-length open reading frame (ORF) of DPH1 was designed to overexpress DPH1. For this article, it is called DPH1 plasmid. Cells were transfected with plasmids or RNA duplexes using Lipofectamine 2000 (Invitrogen) according to the manufacturer's guidelines. The medium was changed to 2\% FBS 1640 after $6 \mathrm{~h}$. Cells were collected at $24 \mathrm{~h}$ and $48 \mathrm{~h}$ after transfection for RNA isolation and protein extraction, respectively.

\section{Luciferase reporter assay}

The 3'UTR of DPH1 was cloned and inserted downstream of a p-MIR-reporter plasmid (Ambion, Austin, TX, USA).To construct a mutated reporter plasmid, the binding site of miR-218-5p was mutated from AGCACA to TCGTGT. Luciferase reporter assays (see our previous study for details [24]) were performed 24 $\mathrm{h}$ after transfection of SW480 cells.

\section{Cell viability assay}

Six hours after transfection, SW480 cells were seeded onto 96-well plates at $5 \times 10^{4}$ cells per well and incubated in 10\% FBS 1640.The numbers of cells were assessed at 12, 24, 36, 48 and60 h using Cell CountingKit-8(CK04-500, Dojindo). $10 \mu \mathrm{L}$ Cell CountingKit-8 solution per well was added into the medium. After incubation for $3 \mathrm{~h}$, absorbance was measured at $450 \mathrm{~nm}$. An EdU proliferation assay was also used to determine cell viability.EdU Cell Proliferation Assay Kit was purchased from Ribobio (Guangzhou, China). Six hours after transfection, SW480 cells were seeded onto 48-well plates. Forty-eight hours later, the cells were incubated with $50 \mu \mathrm{M} 5$-ethynyl-2'-deoxyuridine (EdU) according to the manufacturer's guidelines. After incubation for $8 \mathrm{~h}$, fixation, permeabilization and staining were performed successively. A florescence microscope was used to capture the nucleated cells and to determine the proportion of EdU positive cells.

\section{Cell invasion assay}

Cell invasion assays were performed using 24-well 8- $\mu$ m-pore-sized chambers (BD Biosciences, Bedford, MA, USA)which were coated with a thin layer of Matrigel. Six hours after transfection, SW480 cells were seeded onto the upper chamberat $2 \times 10^{4}$ cells per well with serum-free 1640 media. The lower spaces were added with 10\% FBS 1640.Cells were incubated and allowed to invade for $48 \mathrm{~h}$. After fixing with icecold methanol and staining with $0.1 \%$ crystal violet solution, cells from the upper face of filters were moved with cotton swabs. Different views of $20 \times$ fields were captured from each membrane and the numbers of cells per field were counted.

\section{KARGER}




\section{Statistical analysis}

Each experiment was performed in triplicate and repeated twice. All images are representative of at least three independent experiments. Statistical analysis was performed using the Student's t-test. Data are shown as the mean \pm SE and considered significant if the $\mathrm{P}$ value was $<0.05$ (indicated by $*$ ),$<0.01$ (indicated by ${ }^{* *}$ ) or $<0.001$ (indicated by $* * *$ ).

\section{Results}

\section{DPH1 functions as an oncogene in CRC cells}

We examined DPH1 expression patterns of 13 paired CRC tissues and their corresponding noncancerous tissues. Both the protein and mRNA levels of DPH1 were observed to be dramatically increased in CRC tissues compared with the noncancerous tissues (Fig. 1A -1C).

To determine the effects of DPH1 on CRC cell functions, we knocked down and overexpressed DPH1 in the human CRC cell lines SW480 and HT29. To silence DPH1, small interfering RNAs targeting DPH1 (DPH1 siRNAs) were designed. To overexpress DPH1, a plasmid specifically expressing the full-length DPH1 ORF (DPH1 plasmid) was designed. Effective knockdown or overexpression of DPH1 is shown in Fig. 2A-2B. DPH1 siRNA-3 and DPH1 plasmid were selected for further experiments. We performed CCK-8 and EdU assays to investigate cell proliferation and the transwell invasion assay to investigate cell invasive capability. SW480 cells transfected with DPH1 siRNA-3 showed a decline in cell proliferation (Fig. 2C-2F) and cell invasion (Fig. 2G and 2H), whereas SW480 cells transfected with DPH1 plasmid exhibited an increase in cell proliferation (Fig. 2C-2F) and invasion (Fig. 2G and 2H). These results indicate that DPH1 functions as an oncogene rather than a tumour suppressor in CRC cells.

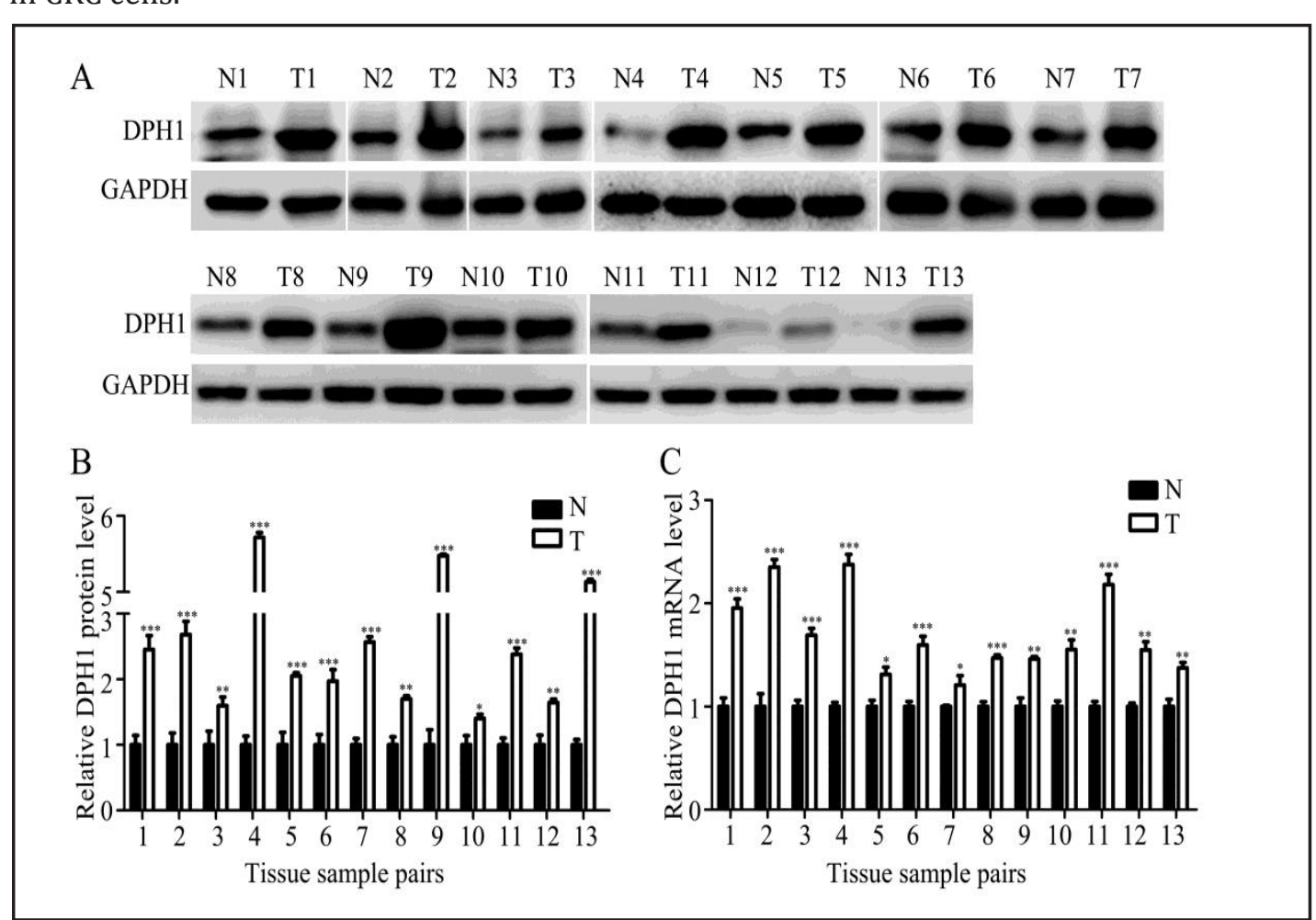

Fig. 1. Both increase of DPH1 protein and mRNA levels in CRC tissues. (A and B) Western blotting analysis of DPH1 protein levels in 13 paired CRC tissues (T) and their corresponding noncancerous tissues (N). A: Representative images. B: Quantitative analysis. (C) Quantitative RT-PCR analysis for the relative DPH1 mRNA levels in the same 13 paired $\mathrm{CRC}$ tissues $(\mathrm{T})$ and their corresponding noncancerous tissues $(\mathrm{N}) .{ }^{* *} \mathrm{P}<0.01$ and $* * * \mathrm{P}<0.001$.

\section{KARGER}


Fig. 2. DPH1 plays an oncogenic role in $\mathrm{CRC}$ cell lines. (A and B) DPH1 protein levels after knockdown and overexpression of DPH1 in SW480 and HT29 cells. A: Representative images. B: Quantitative analysis. (C and D) Cell viability activities of SW480 cells were assessed 12, 24, 36, 48 and $60 \mathrm{~h}$ after transfection with control siRNA and DPH1 siRNA-3, control plasmid and DPH1 plasmid, using Cell Counting Kit-8. (E and F) The EdU proliferation assay was also performed $48 \mathrm{~h}$ after transfection of SW480 cells with control SiRNA, DPH1 siRNA-3, control plasmid and DPH1 plasmid. The cells with red fluorescence are in the $\mathrm{S}$ phase of mitosis, while the cells with blue fluorescence represent all of the cells. E: Representative images. F: Quantitative analy-
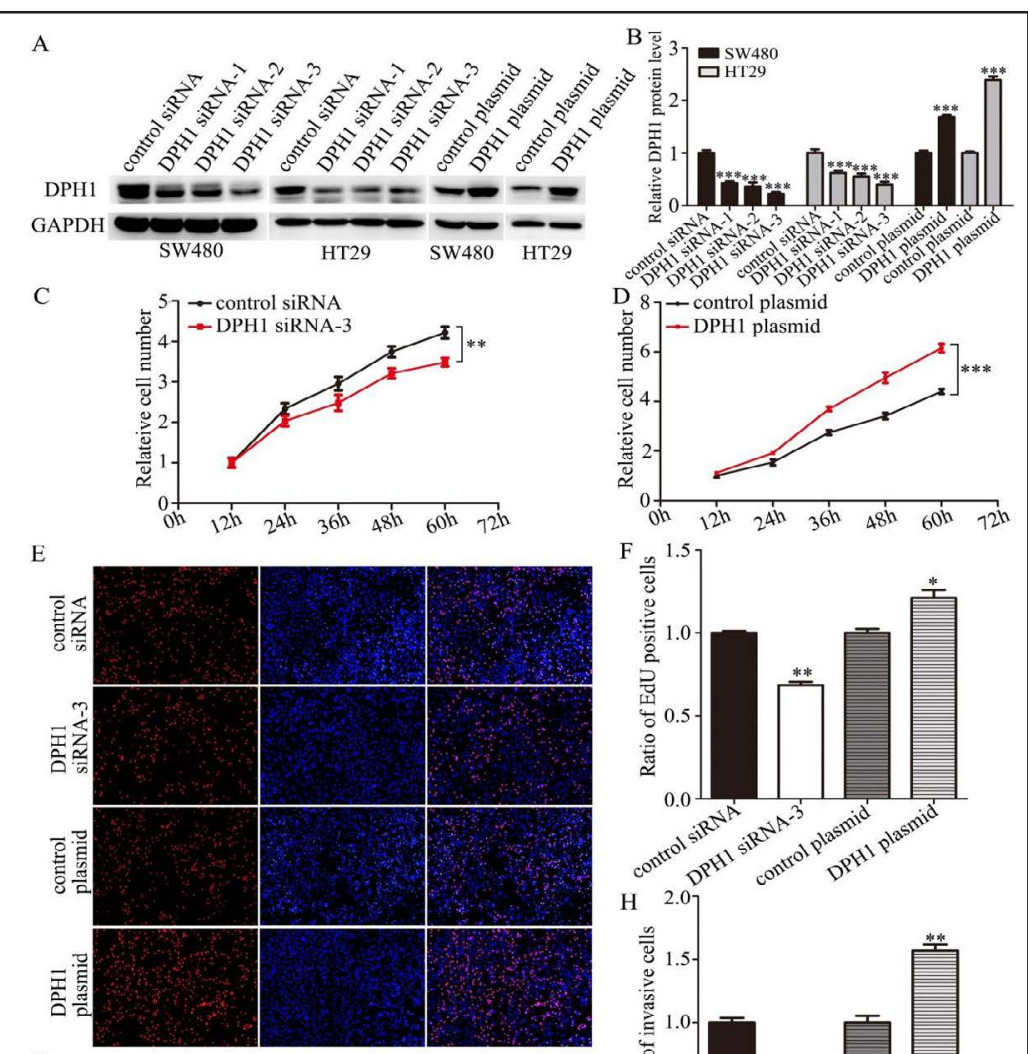

G

control siRNA DPHI siRNA-3 control plasmid DPH1 plasmid control siRNA DPHI SIRNA-3 control plasmid DPH1 plasmid

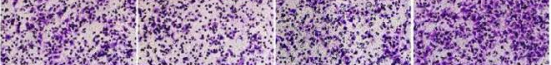

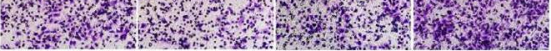
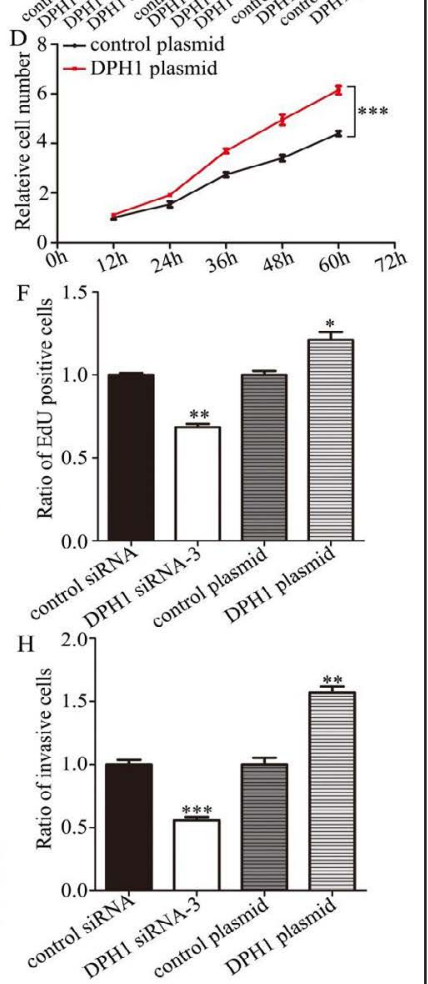

sis of EdU-positive SW480

cells. ( $G$ and H) Cell invasion ability was analysed using transwell assays after transfection of SW480 cells with control siRNA, DPH1 siRNA-3, control plasmid and DPH1 plasmid. G: Representative images. H: Quantitative analysis. ${ }^{*} \mathrm{P}<0.05,{ }^{* *} \mathrm{P}<0.01$ and ${ }^{* * *} \mathrm{P}<0.001$.

\section{Confirmation of DPH1 as a novel target of miR-218-5p}

Next, we determined how DPH1 was upregulated in CRC cells. Because miRNAs are powerful upstream regulators of genes, we wondered whether any miRNAs were involved in DPH1 regulation. Using three publicly available algorithms (TargetScan [13], miRanda [14] and PicTar [15]), miR-218-5p, a well-known tumour suppressor, was predicted as a candidate regulator of DPH1. The illustration of hybridization between miR-218-5p and DPH1 3'UTR is shown in Fig. 3A, the minimum free energy value of which is $-17.2 \mathrm{kcal} / \mathrm{mol}$. Furthermore, the binding sequence is highly conserved across multiple species.

Next, we assessed the levels of miR-218-5p in the CRC and noncancerous tissues using quantitative RT-PCR. Consistent with previous studies, miR-218-5p levels were decreased in CRC tissues compared with noncancerous tissues (Fig. 3B). To examine the correlation between miR-218-5p and DPH1, we evaluated DPH1 expression after either increasing or decreasing miR-218-5p levels in SW480 and HT29 cells using pre-miR-218-5p or antimiR-218-5p. Effective overexpression and knockdown of miR-218-5p is shown in Fig. e 3C. Consequently, the DPH1 mRNA and protein levels were significantly decreased due to miR218-5p overexpression and increased due to miR-218-5p knockdown (Fig. 3D-3F). These results prove that miR-218-5p negatively correlates negatively with DPH1 in CRC cells. 
A

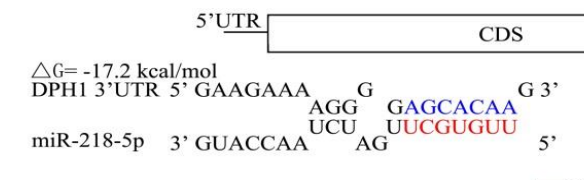

B

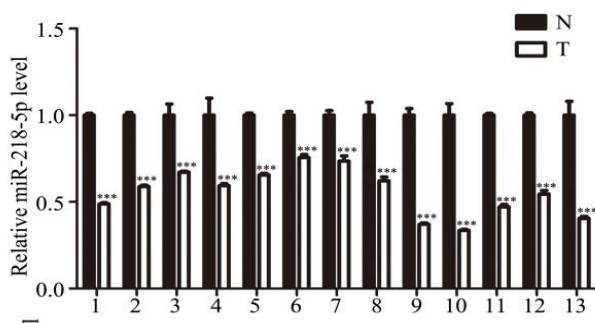

D

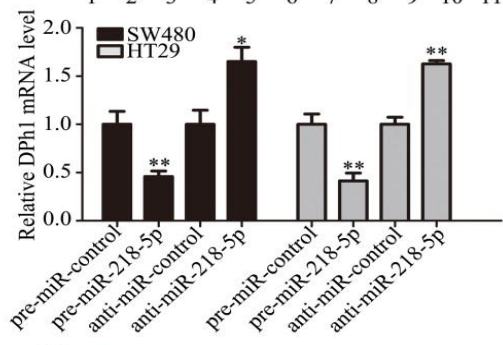

F

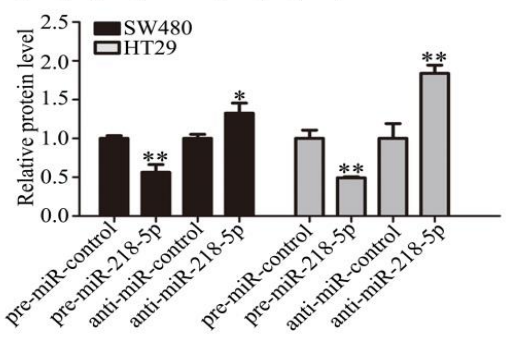

E

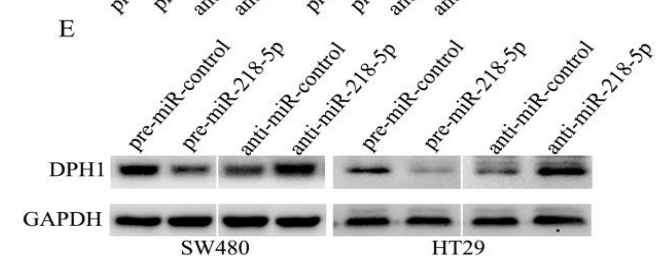

Human GUACUGAAGAAAAGGGGAGCACAAG
Chimp GUACUGAAGAAAAGGGGAGCACAAG
Rhesus GUAUUGAGAAAAGGGAGCACAAG

$\mathrm{C}$

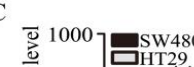

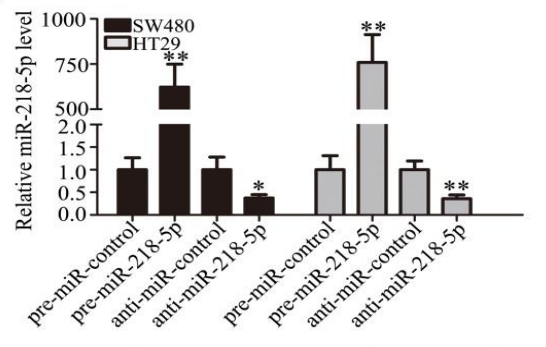

G

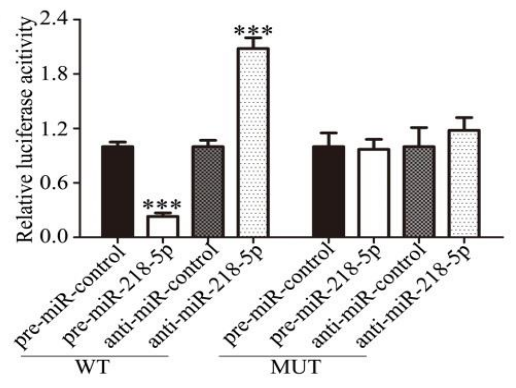

Fig. 3. DPH1 is a target of miR-218-5p. (A) Illustration of hybridization between miR-218-5p and the DPH1 3'UTRbinding site. (B) Relative miR-218-5p levels in 13 pairs of colorectal cancer tissues and their corresponding noncancerous tissues. (C) Relative miR-218-5p levels in SW480 and HT29 cells transfected with pre-miR-control, pre-miR-218-5p, anti-miR-control and anti-miR-218-5p. (D) Relative DPH1 mRNA levels in SW480 and HT29 cells transfected with pre-miR-control, pre-miR-218-5p, anti-miR-control and anti-miR-218-5p. (E and F) DPH1 protein levels in SW480 and HT29 cells transfected with pre-miR-control, pre-miR-218-5p, anti-miR-control and anti-miR-218-5p. E: Representative images. F: Quantitative analysis. (G) Luciferase reporters containing wild-type (WT) or mutant (MUT) recognition site of miR-218-5p were co-transfected into SW480 cells with pre-miR-control, pre-miR-218-5p, anti-miR-control and anti-miR-2185 p. Luciferase activities were assessed and normalized. ${ }^{*} \mathrm{P}<0.05,{ }^{* *} \mathrm{P}<0.01$ and ${ }^{* * *} \mathrm{P}<0.001$.

To determine whether miR-218-5p directly targets DPH1 by binding to the DPH1 mRNA 3'UTR, we performed luciferase reporter assay. As expected, significantly reduced luciferase activity was observed in the cells transfected with pre-miR-218-5p while the activity was increased in the cells treated with anti-miR-218-5p (Fig. 3G).Furthermore, the activity of mutated luciferase reporter was unaffected (Fig. 3G). These results validated that miR-2185 p directly targets DPH1 in CRC.

\section{miR-218-5p suppresses CRC cell proliferation and invasion by regulating DPH1}

We hypothesized that miR-218-5p suppresses CRC carcinogenesis by inhibiting DPH1 expression. Thus, we further analysed the biological effects of DPH1 gene repression by miR218-5p in SW480 cells. As the results show, cells treated with pre-miR-218-5p exhibited reduced cell proliferation (Fig. 4A-4D) and invasion (Fig. 4E and 4F), whereas cells transfected 


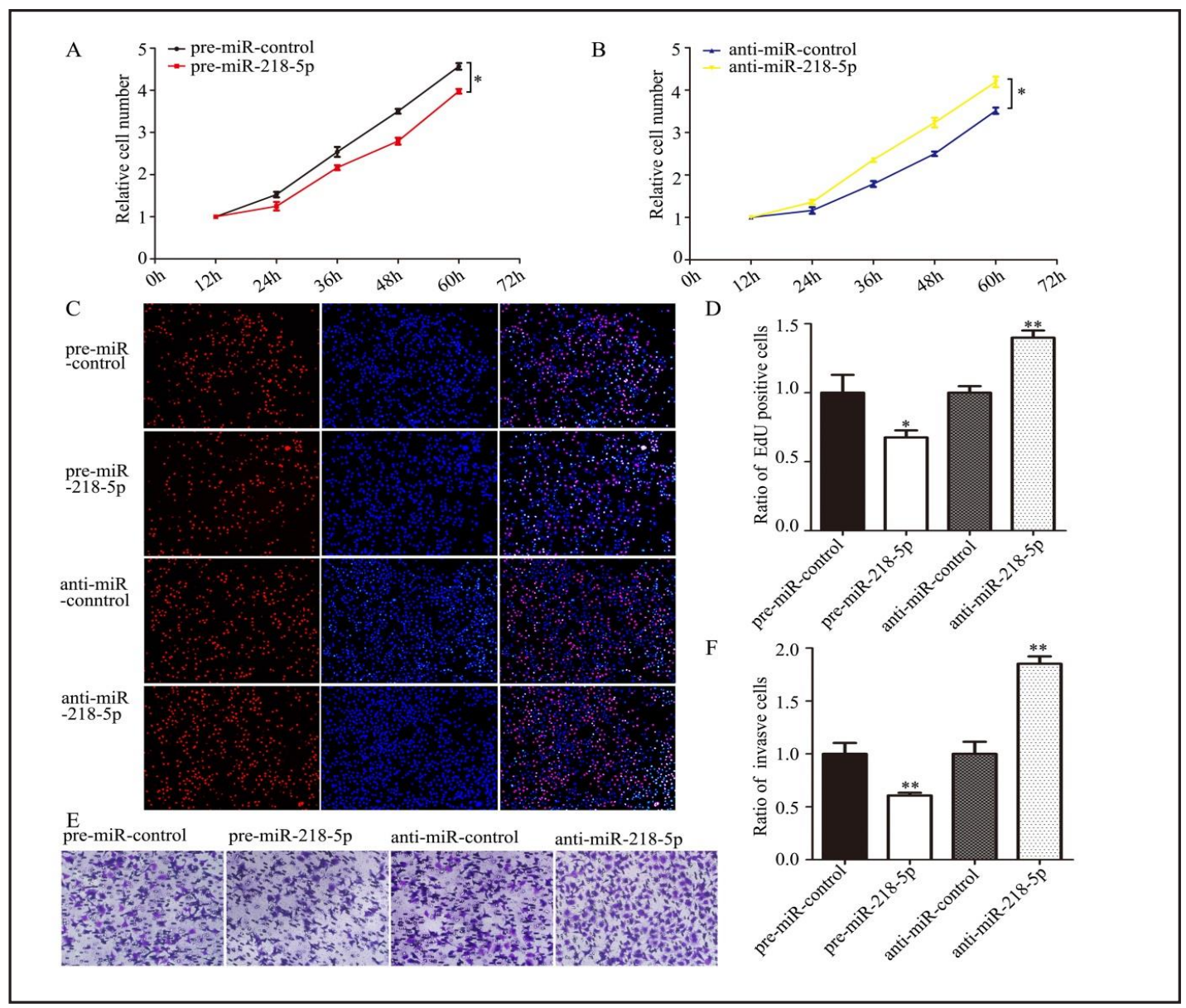

Fig. 4. Effect of miR-218-5p on CRC cell proliferation and invasion. (A and B) Cell viability activities were determined using Cell Counting Kit-8 after transfection of SW480 cells with pre-miR-control, pre-miR-2185p, anti-miR-control and anti-miR-218-5p. (C and D) The EdU proliferation assay was also performed after transfection of SW480 cells with pre-miR-control, pre-miR-218-5p, anti-miR-control and anti-miR-2185p. C: Representative images. D: Quantitative analysis. (E and F) Cell invasion ability was analysed using transwell assays after transfection of SW480 cells with pre-miR-control, pre-miR-218-5p, anti-miR-control and anti-miR-218-5p. E: Representative images. F: Quantitative analysis. ${ }^{*} \mathrm{P}<0.05$ and ${ }^{* *} \mathrm{P}<0.01$.

with anti-miR-218-5p displayed increased cell proliferation (Fig. 4A-4D) and invasion (Fig. $4 \mathrm{E}$ and $4 \mathrm{~F}$ ). We further investigated whether the influences of miR-218-5p on the CRC cells come out of miR-218-5p-driven DPH1 repression. We found thatSW480 cells co-transfected with pre-miR-218-5p andDPH1 plasmid displayed a significantly higher viability activity (Fig. 5A-5C) than cells treated with pre-miR-218-5p, implying that miR-218-5p-resistant DPH1 suffices for reverting the miR-218-5p-driven repression of DPH1 and reducing the anti-proliferative abilities of miR-218-5p in CRC cells. Analogously, we observed that cells co-transfected with pre-miR-218-5p and DPH1 plasmid had higher invasive rates than cells transfected with pre-miR-218-5p alone (Fig. 5D and 5F). These data manifest that miR-2185 pcouldsuppress cell viability and cell invasion through knocking down DPH1.

\section{Discussion}

Many factors contribute to CRC including genetic alterations and epigenetic alterations. In this study, we found that the miR-218-5p/DPH1 axis plays an oncogenic role in CRC by promoting CRC cell viability and cell invasion. 


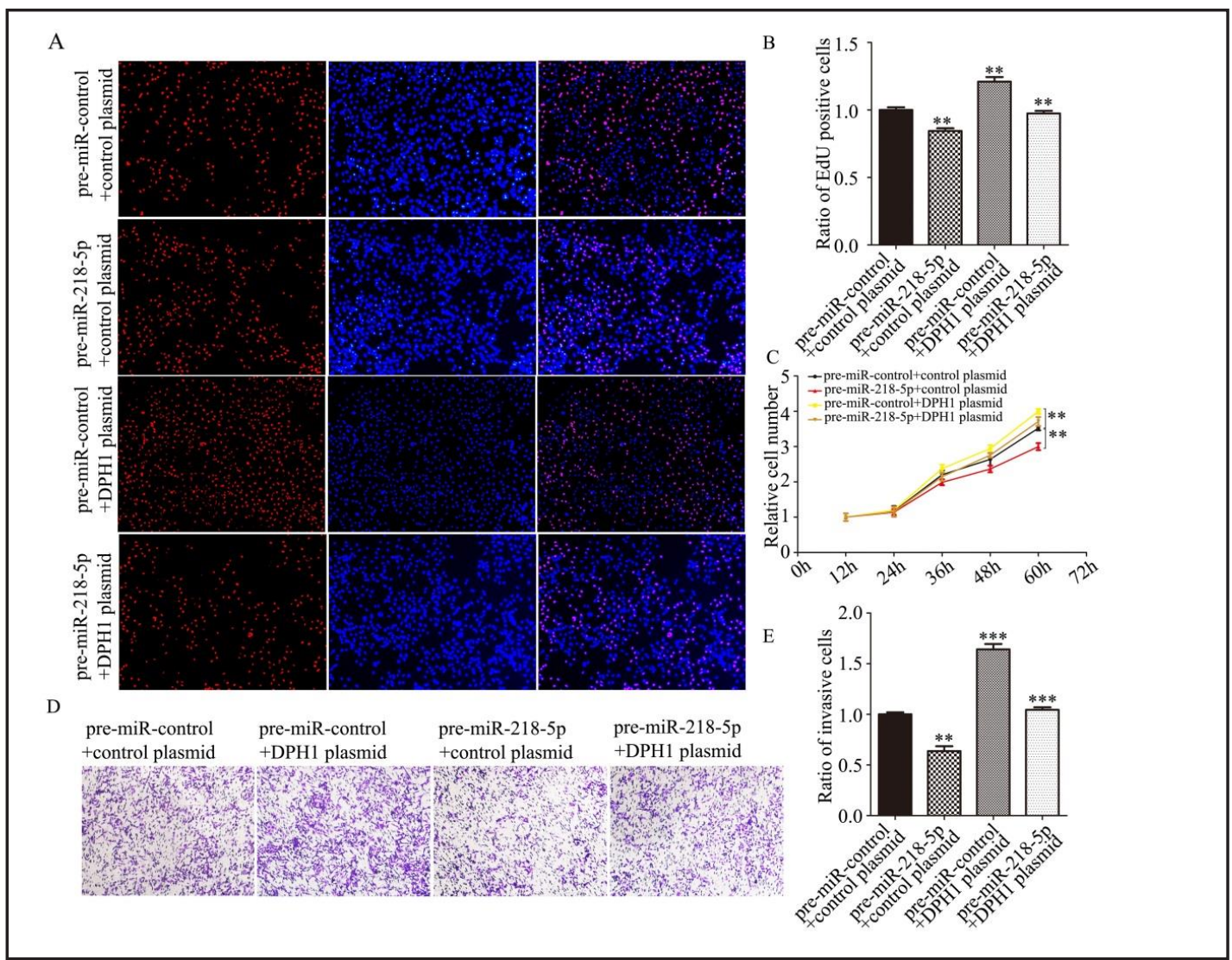

Fig. 5. Co-effect of miR-218-5p and DPH1 on CRC cell proliferation and invasion. (A and B) The EdU proliferation assay was performed after transfection of SW480 cells with pre-miR-control plus control plasmid, pre-miR-control plus DPH1 plasmid, pre-miR-218-5p plus control plasmid and pre-miR-2185p plus DPH1 plasmid. A: Representative images. B: Quantitative analysis. (C) CCK-8 viability assay was performed after transfection of SW480 cells with pre-miR-control plus control plasmid, pre-miR-control plus DPH1 plasmid, pre-miR-218-5p plus control plasmid and pre-miR-218-5p plus DPH1 plasmid. (D and E) Cell invasion ability was analysed using transwell assays after transfection of SW480 cells with pre-miRcontrol plus control plasmid, pre-miR-control plus DPH1 plasmid, pre-miR-218-5p plus control plasmid and pre-miR-218-5p plus DPH1 plasmid. D: Representative images. E: Quantitative analysis. ${ }^{*} \mathrm{P}<0.05,{ }^{* *} \mathrm{P}<0.01$ and $* * * \quad \mathrm{P}<0.001$.

For many years, DPH1 has been known as a putative tumour suppressor in ovarian cancer and breast cancer. In 1999, DPH1 was first reported as a candidate tumour suppressor that inhibited cell growth in ovarian cancer [4]. Years later, a number of studies suggested a tumour suppressive role for DPH1 in ovarian and breast cancers [5, 16]. But DPH1 is ubiquitously expressed in human tissues during various stages of development [17], not just in the ovary and breast. Aberrant DPH1 expression in different areas of the body might result in other diseases. For example, Yu et al. reported that DPH1 deficiency in the neural crest contributes to craniofacial abnormalities in patients with Miller-Dieker syndrome [18]. We found that DPH1 is overexpressed in CRC and correlates with CRC pathogenesis. This is the first report demonstrating the participation of DPH1 in CRC and uncovers another aspect of DPH1 as an oncogene. Given that DPH1 is also involved in diphthamide biosynthesis, its upregulation in CRC may also affect other signalling pathways. These effects therefore require further investigation.

Why DPH1 is upregulated during CRC tumourigenesis is an interesting problem deserving further investigation. Because miRNAs can negatively regulate gene expression, we hypothesized miRNAs may be involved in this process. As a matter of fact, increasing 
evidences have shown that miRNAs are closely related to CRC tumorigenesis. For example, miR-203 and miR-124 inhibits cell proliferation in CRC $[19,20]$. miR-218 plays a vital role in represses cell viability and invasion during CRC development [21]. In this study, through bioinformatics analysis and a series of experiments, we demonstrated that miR-218-5p directly targets DPH1 and showed that loss of miR-218-5p leads to DPH1 upregulation. Consistent with previous studies, we also proved that miR-218-5p inhibits CRC cell viability and cell invasion. Modulation of DPH1 by miR-218-5p explains why DPH1 is aberrantly overexpressed in CRC patients.

In fact, miR-218 targets many different genes, meaning that we cannot eliminate the possibility that additional targets are also influenced by miR-218-5p. For example, miR-218 has been reported to directly repress the oncogenes BRCA1 [22], Survivin [11] and mTOR[23]. Hence, it is significant to research how crucial the newly identified pathway would be in the CRC tumourigenesis network. In this article, we found that restoration of DPH1 expression via a miR-218-5p-resistant DPH1 overexpression plasmid completely reversed miR-218$5 p$-induced cellular phenotypes, suggesting that DPH1 is a dominant target of miR-218-5p. However, the exact contribution of the miR-218-5p-DPH1 axis to CRC tumourigenesis still needs to be investigated further in vivo.

In conclusion, this study is the first to identify that DPH1 functions as an oncogene in CRC and can be negatively modulated by miR-218-5p to promote CRC tumourigenesis. Our findings provide new insights into the biological functions of DPH1 and the molecular mechanisms of CRC carcinogenesis.

\section{Conclusion}

DPH1 function as an oncogene and is regulated by miR-218-5p in CRC cells. The miR218-5p/DPH1 axis plays an important role in the proliferation and invasion of CRC cells.

\section{Acknowledgements}

Grants from the National Basic Research Program of China (973 Program) (No. 2014CB542300)supported this work, along with the National Natural Science Foundation of China (No. 31271378 and No. 81472508), the Research Special Fund for Public Welfare Industry of Health (No. 201302018), and the Natural Science Foundation of Jiangsu Province (No. BE2016737 and No. BK20140601 and No. BK20141376).

\section{Disclosure Statement}

The authors have declared that no Disclosure Statement exists.

\section{References}

1 Siegel RL, Miller KD, Jemal A: Cancer statistics, 2016. CA Cancer J Clin 2016;66:7-30.

-2 Chen W, Zheng R, Baade PD, Zhang S, Zeng H, Bray F, Jemal A, Yu XQ He J: Cancer statistics in China, 2015. CA Cancer J Clin 2016;66:115-132.

- 3 Liu S, Milne GT, Kuremsky JG, Fink GR, Leppla SH: Identification of the proteins required for biosynthesis of diphthamide, the target of bacterial ADP-ribosylating toxins on translation elongation factor 2. Mol Cell Biol 2004;24:9487-9497.

4 Bruening W, Prowse AH, Schultz DC, Holgado-Madruga M, Wong A, Godwin AK: Expression of OVCA1, a candidate tumor suppressor, is reduced in tumors and inhibits growth of ovarian cancer cells. Cancer Res 1999;59:4973-4983. 


\section{\begin{tabular}{ll} 
Cellular Physiology & Cell Physiol Biochem 2017;44:505-514 \\
\hline DoI: 1011159/000485087 & 2017 The Author(s). Published by S. Karger AG, Basel
\end{tabular}

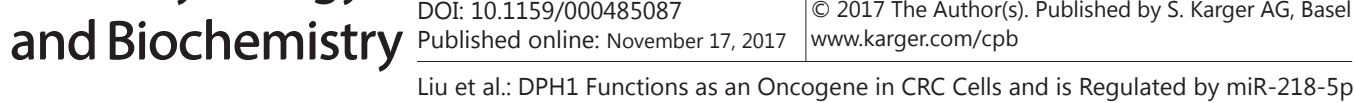

5 Jensen MR, Helin K: OVCA1: emerging as a bona fide tumor suppressor. Genes Dev 2004;18:245-248.

6 He L, Hannon GJ: MicroRNAs: small RNAs with a big role in gene regulation. Nat Rev Genet 2004;5:522-531.

7 Bartel DP: MicroRNAs: genomics, biogenesis, mechanism, and function. Cell 2004;116:281-297.

8 Ilm K, Fuchs S, Mudduluru G, Stein U: MACC1 is post-transcriptionally regulated by miR-218 in colorectal cancer. Oncotarget 2016;7:53443-53458.

-9 Yu H, Gao G, Jiang L, Guo L, Lin M, Jiao X, Jia W, Huang J: Decreased expression of miR-218 is associated with poor prognosis in patients with colorectal cancer. Int J Clin Exp Pathol 2013;6:2904-2911.

10 Zhu K, Ding H, Wang W, Liao Z, Fu Z, Hong Y, Zhou Y, Zhang CY, Chen X: Tumor-suppressive miR-2185 p inhibits cancer cell proliferation and migration via EGFR in non-small cell lung cancer. Oncotarget 2016;7:28075-28085.

11 Hu Y, Xu K, Yague E: miR-218 targets survivin and regulates resistance to chemotherapeutics in breast cancer. Breast Cancer Res Treat 2015;151:269-280.

12 He X, Dong Y, Wu CW, Zhao Z, Ng SS, Chan FK, Sung JJ, Yu J: MicroRNA-218 inhibits cell cycle progression and promotes apoptosis in colon cancer by downregulating BMI1 polycomb ring finger oncogene. Mol Med 2013;18:1491-1498.

13 Lewis BP, Shih IH, Jones-Rhoades MW, Bartel DP, Burge CB: Prediction of mammalian microRNA targets. Cell 2003;115:787-798.

14 Krek A, Grun D, Poy MN, Wolf R, Rosenberg L, Epstein EJ, MacMenamin P, da Piedade I, Gunsalus KC, Stoffel M, Rajewsky N: Combinatorial microRNA target predictions. Nat Genet 2005;37:495-500.

15 John B, Enright AJ, Aravin A, Tuschl T, Sander C, Marks DS: Human MicroRNA targets. PLoS Biol 2004;2:e363.

16 Chen CM, Behringer RR: Ovca1 regulates cell proliferation, embryonic development, and tumorigenesis. Genes Dev 2004;18:320-332.

17 Chen CM, Behringer RR: Cloning, structure, and expression of the mouse Ovca1 gene. Biochem Biophys Res Commun 2001;286:1019-1026.

18 Yu YR, You LR, Yan YT, Chen CM: Role of OVCA1/DPH1 in craniofacial abnormalities of Miller-Dieker syndrome. Hum Mol Genet 2014;23:5579-5596.

19 Wei W, Yang Y, Cai J, Cui K, Li RX, Wang H, Shang X, Wei D: MiR-30a-5p Suppresses Tumor Metastasis of Human Colorectal Cancer by Targeting ITGB3. Cell Physiol Biochem 2016;39:1165-1176.

20 Zhou L, Xu Z, Ren X, Chen K, Xin S: MicroRNA-124 (MiR-124) Inhibits Cell Proliferation, Metastasis and Invasion in Colorectal Cancer by Downregulating Rho-Associated Protein Kinase 1(ROCK1). Cell Physiol Biochem 2016;38:1785-1795.

21 Zhang X, Shi H, Tang H, Fang Z, Wang J, Cui S: miR-218 inhibits the invasion and migration of colon cancer cells by targeting the PI3K/Akt/mTOR signaling pathway. Int J Mol Med 2015;35:1301-1308.

22 He X, Xiao X, Dong L, Wan N, Zhou Z, Deng H, Zhang X: MiR-218 regulates cisplatin chemosensitivity in breast cancer by targeting BRCA1. Tumour Biol 2015;36:2065-2075.

23 Uesugi A, Kozaki K, Tsuruta T, Furuta M, Morita K, Imoto I, Omura K, Inazawa J: The tumor suppressive microRNA miR-218 targets the mTOR component Rictor and inhibits AKT phosphorylation in oral cancer. Cancer Res 2011;71:5765-5778.

24 Liu M, Yang R, Urrehman U, Ye C, Yan X, Cui S, Hong Y, Gu Y, Liu Y, Zhao C, Yan L, Zhang CY, Liang H, Chen X: MiR-19b suppresses PTPRG to promote breast tumorigenesis. Oncotarget 2016;7:64100-64108. 